\title{
Monotonicity-preserving bootstrapped Kriging metamodels for expensive simulations
}

\author{
JPC Kleijnen $^{1 *}$ and WCM van Beers ${ }^{2}$ \\ ${ }^{I}$ Tilburg University, Tilburg, the Netherlands; and ${ }^{2}$ University of Amsterdam, Amsterdam, the Netherlands
}

Kriging metamodels (also called Gaussian process or spatial correlation models) approximate the Input/ Output functions implied by the underlying simulation models. Such metamodels serve sensitivity analysis, especially for computationally expensive simulations. In practice, simulation analysts often know that this Input/Output function is monotonic. To obtain a Kriging metamodel that preserves this characteristic, this article uses distribution-free bootstrapping assuming each input combination is simulated several times to obtain more reliable averaged outputs. Nevertheless, these averages still show sampling variation, so the Kriging metamodel does not need to be an exact interpolator; bootstrapping gives a noninterpolating Kriging metamodel. Bootstrapping may use standard Kriging software. The method is illustrated through the popular $\mathrm{M} / \mathrm{M} / 1$ model with either the mean or the $90 \%$ quantile as output; these outputs are monotonic functions of the traffic rate. The empirical results demonstrate that monotonicity-preserving bootstrapped Kriging gives higher probability of covering the true outputs, without lengthening the confidence interval.

Journal of the Operational Research Society (2013) 64, 708-717. doi:10.1057/jors.2011.148

Published online 22 August 2012

Keywords: simulation; sensitivity analysis; Kriging; bootstrapping; queueing

\section{Introduction}

Monotonicity is a known characteristic of many realistic simulation models. For example, supply chains consist of a sequence of waiting lines and inventories and have decision variables that are known to have positive effects on waiting (queueing) times and service (fill) rates; that is, higher traffic rates increase waiting times, and higher inventory levels increase service rates (simulation is needed to estimate the sizes of these effects). A case study taking advantage of monotonicity is the simulation of a supply chain at Ericsson in Sweden (Kleijnen, 2009). According to a classic simulation textbook (Law, 2007, pp 12-47), a building block of realistic simulation models - such as supply chains - is the $\mathrm{M} / \mathrm{M} / 1$ queueing model; in this article we experiment with this basic simulation model. It is relatively easy to derive analytically that in $\mathrm{M} / \mathrm{M} / 1$ models the traffic rate increases the mean steady-state waiting time monotonically (see Law, 2007, pp 79-83).

Note: Monotonicity also plays a role in the following problems. Searching for the short list of really important factors among the many potentially important factors in a large realistic simulation model may be done through a statistical screening technique known as 'sequential

\footnotetext{
${ }^{*}$ Correspondence: JPC Kleijnen, Department of Information Management/Center, Tilburg University, Postbox 90153, 5000 LE Tilburg, the Netherlands.

E-mail: Kleijnen@tilburguniversity.edu
}

bifurcation', which assumes a monotonic Input/Output (I/O) function (Kleijnen, 2009). An example of monotonicity outside simulation is medical research where the response is often assumed to be a monotonic function of the medicine dose (Frazier et al, 2009).

Because monotonicity of $\mathrm{I} / \mathrm{O}$ functions of real or simulated systems may be an important property, monotonicity is also of common interest in mathematical statistics such as regression analysis (namely, in 'iso-tonic' regression and 'rank' regression; see Kleijnen, 2008, pp 98, 162), artificial neural networks (Velikova, 2006), and data mining (Feelders, 2000). In this contribution, however, we focus on Kriging (Gaussian Process modelling, spatial correlation modelling) for the statistical analysis of simulation models with monotonic $\mathrm{I} / \mathrm{O}$ functions. Monotonicity preservation of Kriging metamodels is also examined by Siem (2007), but he does not succeed in finding a solution.

Kriging has already acquired a track record in deterministic simulation of engineering systems; see the classic article on Kriging in deterministic simulation (Sacks et al, 1989), the popular textbook (Santner et al, 2003) and the additional recent references in Kleijnen (2008, p 3). Recently, Kriging is also becoming the topic of research in random (stochastic) simulation-especially discrete-event simulation - which is very popular in Operational Research (OR); see Yin et al (2009) and Ankenman et al (2010). In both deterministic and random simulation, 
the goal of Kriging is to estimate a metamodel (response surface, surrogate, emulator), which approximates the $\mathrm{I} / \mathrm{O}$ function implied by the simulation model at hand. The goal of a metamodel is (i) sensitivity analysis - either global ('what if' analysis) or local (gradient or derivative estimation) - of the simulation model; (ii) verification and validation of the simulation model; (3) optimization of the real system being simulated. We focus on global sensitivity analysis, which is the basis of the other goals.

To estimate (calibrate) a metamodel (eg, a Kriging model), the simulation analysts must experiment with the simulation model; that is, they must run the simulation model with different combinations of the values for the inputs of the simulation model. Unfortunately, practical simulation models may be expensive; that is, these models may require much computer time to obtain the output (say) $w$ for an input combination $\mathbf{x}$; for example, it took 36 to $160 \mathrm{~h}$ of computer time to simulate a car-crash model at Ford (Simpson et al, 2004; Ankenman et al, 2010). The analysts must then fit a metamodel to a relatively small number (say) $n$ of input combinations $\mathbf{x}_{i}$ $(i=1, \ldots, n)$ actually simulated. Unfortunately, these $n$ input combinations replicated $m_{i}$ times may be so small that the 'classic' Kriging metamodel does not preserve the monotonic shape of the $\mathrm{I} / \mathrm{O}$ function, but shows wiggling (erratic) behaviour. The main contribution of this article is to derive bootstrapped Kriging that avoids this wiggling. (If $n$ and $m_{i}$ are 'large', then classic Kriging does not violate the monotonicity assumed for the underlying simulation's $\mathrm{I} / \mathrm{O}$ function so there is no need for our bootstrapped Kriging.)

Mathematically speaking, a Kriging model is an exact interpolator; that is, the Kriging predictions equal the outputs observed for the $n$ 'old' (already simulated) input combinations. In stochastic simulation (with its random outputs) this interpolation property is not desirable, so the Kriging metamodel is slightly changed; for details we refer to the literature (Santner et al, 2003, pp 215-249; Forrester et al, 2008, p 143; Yin et al, 2009; Ankenman et al, 2010). This 'stochastic Kriging' does not interpolate the $n$ outputs averaged over the (say) $m_{i}(i=1, \ldots, n)$ replicates for input combination $i$ (these replicates are Identically, Independently Distributed or IID if they use nonoverlapping Pseudo-Random Number or PRN streams). Recently, Chen et al (2010) also account for correlations between the outputs for different input combinations caused by the use of Common Random Numbers (CRN); by definition, CRN use the same PRN seed (initial value) to generate the same replication number (say) $r$ for all the $n$ input combinations with $r=1, \ldots$, $m=\min _{i} m_{i}$ (we shall return to CRN).

Unfortunately, there is no well-documented software implementing this stochastic Kriging; popular software (either commercial or academic) assumes deterministic simulation. In this article, we focus on the free Matlab
Kriging toolbox called $D A C E$, which is well documented (Lophaven et al, 2002) and is often applied in practice.

Furthermore, we assume a given design - so the $n$ input combinations and their number of replicates $m_{i}$ are giventhat is so small (because the simulation model is so expensive) that the 'classic' Kriging metamodel does not preserve the shape of the $\mathrm{I} / \mathrm{O}$ function, but shows wiggling behaviour. To solve this problem, we shall derive bootstrapped Kriging with the following properties:

(1) Our Kriging shows monotonic behaviour like the $\mathrm{I} / \mathrm{O}$ function of the given simulation model; the simulation analysts and their clients assume that the $\mathrm{I} / \mathrm{O}$ function of that simulation model is monotonic.

(2) Our Kriging is not an exact interpolator; that is, its predictor for the $n$ old input combinations does not necessarily equal the $n$ average simulated outputs $\overline{w_{i}}=\sum_{r=1}^{m_{i}} w_{i ; r} / m_{i}$ (where $m_{i}$ still denotes the number of replicates for input combination $i$, and $i=1, \ldots, n$ ).

(3) Our Kriging also gives a (say) $90 \%$ confidence interval for the predictor.

(4) Our Kriging uses distribution-free bootstrapping of the original simulation outputs and distribution-free confidence intervals (our $\mathrm{M} / \mathrm{M} / 1$ example will show that the normal distribution may be an unrealistic assumption for the simulation output).

(5) Our Kriging accounts for variance heterogeneity of the simulation outputs (so $\operatorname{var}\left(w_{i}\right)$ is an unknown function of $\mathbf{x}_{i}$ ).

Monotonicity-preserving Kriging implies that the estimated gradients should remain positive as the input increases. This monotonicity preservation implies sensitivity analysis results that are understood and accepted by the clients of the simulation analysts so the decision-makers have more confidence in the simulation as a decision support tool. Furthermore, we conjecture that estimated gradients with correct signs will improve simulation optimization - but in this article we do not examine such optimization. We do investigate whether our monotonicity-preserving Kriging gives 'better' predictions than classic Kriging does; that is, we compare the Mean Squared Error (MSE) - which is the standard criterion in Kriging - and the coverage and width of the confidence intervals.

Technically, we realize our Kriging (with the five properties listed above) through 'distribution-free bootstrapping' that we adapt for random simulation. This bootstrapping is conceptually simple: it resamples - with replacement - the replicated simulation outputs. It is computationally inexpensive (requiring micro-seconds), compared with the computer time required by many practical simulations (hours).

Note: Traditionally, bootstrapping is used to estimate the variability of some statistic; for example, estimate the 
true variance of the (nonlinear) Kriging predictor (accounting for the estimation of the Kriging parameters) through parametric bootstrapping assuming a Gaussian process for the deterministic simulation output (Den Hertog et al, 2006). Our bootstrap, however, has a very different goal, namely monotonicity preservation. If the simulation model were deterministic (as in engineering but not in OR), then there would be no replicates so distribution-free bootstrapping would not apply and we would resort to parametric bootstrapping assuming a Gaussian process.

Our main conclusion is that our bootstrap Kriging gives confidence intervals with higher coverage and acceptable length, if the simulation is so expensive that sample sizes are so small that classic Kriging gives nonmonotonic behaviour.

The remainder of this article is organized as follows. First, we detail our monotonicity-preserving bootstrapped Kriging. Next we illustrate this Kriging through the $\mathrm{M} / \mathrm{M} / 1$ simulation model with as output the transientstate average and $90 \%$ quantile of the waiting time in queue. We then present conclusions and topics for further research. Supplementary material is published in an Online Appendix.

\section{Monotonicity-preserving bootstrapped Kriging}

In deterministic simulation, Kriging is an exact interpolator:

$$
y\left(\mathbf{x}_{i}\right)=w\left(\mathbf{x}_{i}\right) i=1, \ldots, n,
$$

where $y$ denotes the Kriging predictor, $w$ the simulation output, $\mathbf{x}_{i}$ the $i$ th combination of the (say) $k \geqslant 1$ simulation inputs with $i=1, \ldots, n$ where $n$ denotes the number of old input combinations that have already been simulated; outputs are predicted through the Kriging metamodel fitted to the $n \mathrm{I} / \mathrm{O}$ combinations.

Random simulation gives different outputs at the same $\mathbf{x}_{i}$ whenever the PRN seed changes. We assume that the simulation analysts obtain $m_{i} \geqslant 2$ replicates; otherwise, they cannot evaluate the variability of the simulation model's output. Moreover we assume that these replicates are IID, because they use nonoverlapping PRN streams and the PRNs are assumed to be IID on the interval $(0,1)$. These replicates enable the following classic unbiased variance estimators:

$$
s_{i}^{2}=\frac{\sum_{r=1}^{m_{i}}\left(w_{i ; r}-\overline{w_{i}}\right)^{2}}{m_{i}-1} \text { with } m_{i} \geqslant ; 2 .
$$

We assume that the simulation model is expensive so $n$ and $m_{i}$ are so small that the fitted Kriging metamodel does not necessarily preserve the monotonicity of the $\mathrm{I} / \mathrm{O}$ function defined by the underlying simulation model; an example is Figure 1 (further discussed below). We assume that if the analysts require monotonicity for the simulation model's I/O function, then they obtain so many replicates that the $n$ average simulation outputs also show this

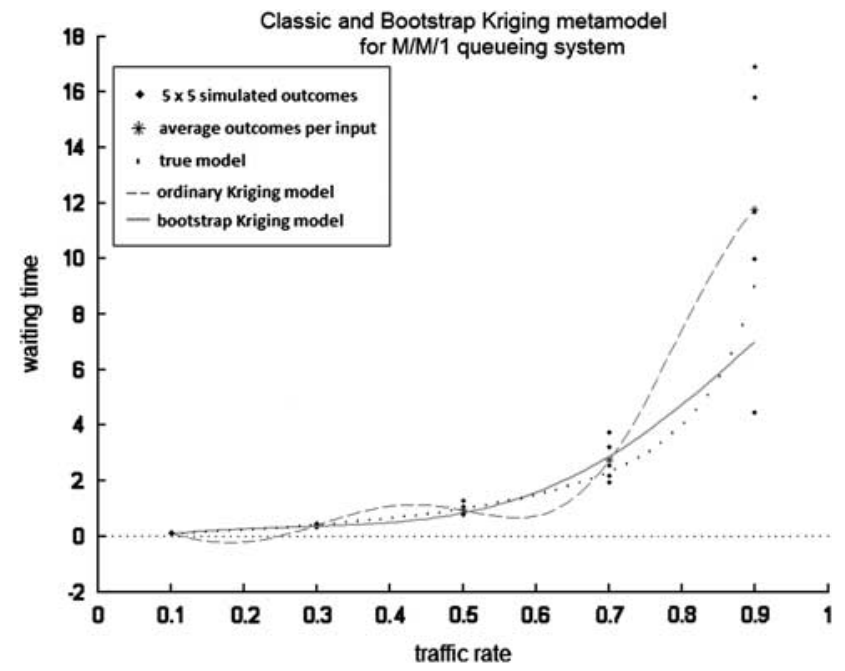

Figure 1 Classic and monotonicity-preserving bootstrapped Kriging metamodels and true $\mathrm{I} / \mathrm{O}$ function for $\mathrm{M} / \mathrm{M} / 1$ with $n=5, m=5, T=1000$.

property; see again Figure 1 for $\mathrm{M} / \mathrm{M} / 1$ with its single input; for multiple inputs see (5) and the $(s, S)$ example. This assumption is realistic if in practice the clients consider the simulation model to be wrong (not valid) if this model generates $\overline{w_{i}}>\overline{w_{i+1}}$ (higher average waiting time for lower traffic rate). Technically, the requirement $\overline{w_{i}}<\overline{w_{i+1}}$ is too strict; that is, the analysts may point out that though the simulation averages are nonmonotonic, the confidence intervals may overlap so they do not violate the monotonicity assumption. Moreover, our monotonicitypreserving bootstrap Kriging procedure has a weaker requirement; namely, $\min _{i} w_{i}<\max _{i} w_{i+1}$, as we shall see below.

Obviously, random simulation gives average outputs $\overline{w_{i}}$ that are still random. Therefore we do not require our Kriging predictor to equal the observed average outputs, whereas we did in previous publications (Van Beers and Kleijnen, 2003; Kleijnen et al, 2010).

A simple data-driven way to account for the randomness of $w_{i ; r}$ is distribution-free (non-parametric) bootstrapping; see the classic textbook on bootstrapping (Efron and Tibshirani, 1993) and the additional recent references in Kleijnen (2008, p 81). This bootstrapping assumes that all $n$ old points are replicated 'enough' times: $m \gg 2$ (in the $\mathrm{M} / \mathbf{M} / 1$ example, $m_{i}=5$ ). So we resample - with replacement - the $m_{i}$ replicates per point $\mathbf{x}_{i}$, which gives the bootstrap observations $w_{i: r}^{*}$ which in turn give the average output $\bar{w}_{i}^{*}$ (the superscript ${ }^{*}$ is the usual symbol for bootstrapped observations). The simulation outputs $w_{i ;}$. have different means and variances at different points $\mathbf{x}_{i}$, so they are not IID (eg, the simulated steady-state mean waiting time has a mean and variance that increases with the traffic rate). We formalize our bootstrap procedure through the following pseudo-code, assuming that no 
$\mathrm{CRN}$ are used (also see the last paragraph of this section) and allowing a possibly non-constant number of replicates, $m_{i}(i=1, \ldots, n)$ :

(1) Initialize the simulation input combination: $i=1$.

(2) Initialize the replicate number: $r=1$.

(3) Resample - with replacement - a replicate number $r^{*}$ from the uniform distribution defined on the integers $1, \ldots, m_{i}$; that is, the uniform density function is $p\left(r^{*}\right)=1 / m_{i}$ with $r^{*}=1, \ldots, m_{i}$.

(4) Replace the $r$ th 'original' output $w_{i ; r}$ by the bootstrap output $w_{i, r}^{*}=w_{i, r^{*}}$ (the original outputs $w_{i ; r}$ are resampled with replacement, which gives $w_{i: r}^{*}=w_{i ; r}^{*}$ ).

(5) If $r<m_{i}$ then $r=r+1$ and return to Step 3; else proceed to the next step (bootstrapping uses the same sample size as the original simulation).

(6) If $i<n$ then $i=i+1$ and return to Step 2; else proceed to the next step.

(7) Compute the Kriging predictor $y^{*}$ from the bootstrapped I/O data set $\left(\mathbf{X}, \overline{\mathbf{w}}^{*}\right)$ where $\mathbf{X}$ denotes the $n \times k$ matrix with the $n$ old combinations of the $k$ simulation inputs and $\overline{\mathbf{w}}^{*}$ denotes the $n$-dimensional vector with the bootstrap averages $\bar{w}_{i}^{*}=\sum_{r=1}^{m_{i}} w_{i, r}^{*} / m_{i}$ and $i=1, \ldots, n$.

To these $\left(\mathbf{X}, \overline{\mathbf{w}}_{i}^{*}\right)$ (the bootstrapped I/O data resulting from Step 7) we fit an interpolating Kriging model $y^{*}$ (analogous to (1)):

$$
y_{i}^{*}=\bar{w}_{i}^{*}(i=1, \ldots, n) .
$$

We point out that we do not fit the Kriging model to an individual output $w_{i: r}^{*}$ because an individual output is noisier.

Kriging for deterministic simulation uses the covariance matrix (say) $\boldsymbol{\Gamma}$ (defined in the Online Appendix, which gives basic Kriging formulas); this $\boldsymbol{\Gamma}$ depends on the correlation parameters $\theta_{j}$ (also defined in this appendix). This $\boldsymbol{\Gamma}$ is usually estimated through Maximum Likelihood Estimation (MLE). To compute these estimates, we use the DACE toolbox. Although we use DACE, we do not assume normality when bootstrapping; we accept this inconsistency because we use DACE's MLE only to estimate the nuisance parameters collected in $\boldsymbol{\Gamma}$. Finally, when we use DACE to estimate $\boldsymbol{\Gamma}$, we assume the popular so-called Gaussian correlation function

$$
\rho_{j}=\exp \left[-\theta_{j} h_{j}^{2}\right],
$$

where $h_{j}=\left|x_{i ; j}-x_{i^{\prime} ; j}\right|$ denotes the Euclidean distance between the values of input $j$ in the input combinations $i$ and $i^{\prime}(j=1, \ldots, k ; i=1, \ldots, n)$ (also see the Online Appendix).

Because of the randomness in bootstrapping, the resampling is repeated (say) $B$ times; $B$ is called the 'bootstrap sample size'. The value for $B$ is selected rather arbitrarily - a typical choice is $B=100$ - but after observing the results for $B$ bootstrap samples, more samples might be selected if deemed necessary - as we shall see. So (3) results in $B$ bootstrapped Kriging predictors $\mathbf{y}_{b}^{*}=\left(y_{1: b}^{*}, \ldots, y_{n: b}^{*}\right)^{\prime}$ with $b=1, \ldots, B$; the bootstrapped predictor $\mathbf{y}_{b}^{*}$ uses the MLE (say) $\widehat{\theta_{j ; b}}{ }^{*}$ computed from $\left(\mathbf{X}, \overline{\mathbf{w}}_{i}^{*}\right)$; also see (4).

From these $B$ predictors we select the (say) $B^{\prime}(\leqslant B)$ strictly monotonically increasing ones (we select $B^{\prime}$ such that reasonable confidence intervals result; see below) and throw away the remaining ones. Let $\mathbf{x}_{i}<\mathbf{x}_{i^{\prime}}$ denote that at least one component of $\mathbf{x}_{i}$ is smaller than the corresponding component of $\mathbf{x}_{i^{\prime}}$ and none of the remaining components is bigger $\left(i, i^{\prime}=1, \ldots, n\right)$; for example, the $\mathbf{M} / \mathbf{M} / 1$ example with $k=1$ implies that $\mathbf{x}_{i}<\mathbf{x}_{i^{\prime}}$ becomes $x_{i}<x_{i^{\prime}}$, whereas the $(s, S)$ example with $k=2$ implies that $\mathbf{x}_{i}<\mathbf{x}_{i^{\prime}}$ may mean $s_{i}<S_{i^{\prime}}$ and $S_{i} \leqslant S_{i^{\prime}}$. Kriging in simulation implies that each of the $k$ inputs is measured on a quantitative scale such that the Euclidean distance $h$ featuring in (4) is defined. Most designs in simulation use Latin Hypercube Sampling (LHS), which implies that each of the $k$ inputs has $n$ distinct values that are either exactly or approximately equally spaced; see Kleijnen (2008, pp 126-130). We then get

$$
\begin{aligned}
& y_{i ; b^{\prime}}^{*}<y_{i^{\prime} ; b^{\prime}}^{*} \text { if } \mathbf{x}_{i}<\mathbf{x}_{i^{\prime}} \\
& \quad\left(i, i^{\prime}=1, \ldots, n\right)\left(b^{\prime}=1, \ldots, B^{\prime}\right) .
\end{aligned}
$$

Obviously, we may define 'monotonically decreasing' in a strictly analogous way. Notice that (5) implies that all $k$ components of the $n$ gradients are positive; we denote this by

$$
\nabla y_{i ; b^{\prime}}^{*}>\mathbf{0}(i=1, \ldots, n)\left(b^{\prime}=1, \ldots, B^{\prime}\right) .
$$

These gradients are provided 'for free' by DACE; see the Online Appendix and also the example in Kleijnen (2008, p 143).

So there are $B^{\prime}$ bootstrapped monotonicity-preserving Kriging predictors $y_{b^{\prime}}^{*}\left(b^{\prime}=1, \ldots, B^{\prime}\right)$. We use these predictors to compute $B^{\prime}$ predictions $y_{u: b^{\prime}}^{*}$ for the new input combination $\mathbf{x}_{u}$; we consider $v$ new combinations, so $u=1, \ldots, v$. Thus, we predict not a single new point $\mathbf{x}_{n+1}$ but $v \gg 1$ new points; such a test set is also used in the Kriging literature (Sacks et al, 1989). From the $B^{\prime}$ predictions for point $u$ we compute as the point estimate $y_{u ;\left(\left[0.50 B^{\prime}\right\rceil\right)}^{*}$ where $\lceil x\rceil$ denotes the integer resulting from rounding $x$ upwards and the subscript () denotes the order statistics; so $y_{u:\left(\left[0.50 B_{s}\right]\right)}^{*}$ denotes the sample median (the sample median is not sensitive to outliers, whereas the sample mean is; quantiles such as the median will also be discussed in Equation (9) for our $\mathrm{M} / \mathrm{M} / 1$ simulation).

Note: Instead of the sample median $y_{u ;\left(\left[0.50 B^{\prime}\right]\right)}^{*}$, we might use the sample mean $\overline{y_{u}^{*}}=\sum y_{u: b^{\prime}}^{*} / B^{\prime}$ especially when using Kriging for optimization that uses the resulting explicit function. 
Besides this point estimator $y_{u:\left(\left[0.50 B^{\prime}\right]\right)}^{*}$, we compute the lower and upper bounds of the (say) $90 \%$ confidence interval for the bootstrapped Kriging predictor for the true value at test point $\mathbf{x}_{u}$; namely, $y_{u ;\left(\left[0.50 B^{\prime}\right]\right)}^{*}$ and $y_{u ;\left(\left[0.95 B^{\prime}\right\rceil\right)}^{*}$ where $\lceil x\rceil$ denotes the integer resulting from rounding $x$ downwards; more reined procedures are discussed in Efron and Tibshirani (1993). If this confidence interval turns out to be too wide, then we increase $B^{\prime}$ by increasing the bootstrap sample size $B$; for example, in our $\mathrm{M} / \mathrm{M} / 1$ example we start with $B=100$ but augment $B$ with 100 until either $B^{\prime} \geqslant 100$ or (to avoid excessive computational time) $B=1000$. (Increasing the bootstrap sample size $B$ does not take much computer time compared with increasing the number of simulation replicates $m_{i}$ with $i=1, \ldots, n$.)

Confidence intervals are also given by the classic Kriging literature (Lophaven et al, 2002, p 4; Santner et al, 2003, $\mathrm{p}$ 96). However, this literature assumes normality and computes an estimate of the variance $\widehat{\sigma}_{y}^{2}$ of the classic predictor $y$ that ignores the random character of the Kriging weights resulting from estimating the parameters $\theta_{j}$ in the Kriging correlation functions (4). This estimator is misleading in deterministic simulation (Den Hertog et al, 2006), and we shall present similar results in our examples.

Of course, any confidence interval would have a perfect coverage of $100 \%$ if its width were infinite - but such an interval is useless. We therefore estimate both the coverage and the width of the confidence interval for bootstrapped and classic Kriging - averaged over all $v$ test points - in our examples.

Some authors (Ankenman et al, 2010) recommend not using CRN, because this technique increases the MSE under their assumptions. We, however, claim that the use of CRN should depend on the goal of the metamodel; see again the Introduction and Chen et al (2010). Actually, these goals are related; for example, the estimated gradient (a local sensitivity measure) may be used to estimate the optimum. Because we wish to preserve monotonicity, we recommend avoiding CRN, as CRN reduces the variability of $\overline{\mathbf{w}}_{i}^{*}$, whereas finding a monotonicitypreserving bootstrapped Kriging model is more likely when the $\overline{\mathbf{w}}_{i}^{*}$ have larger variability. Anyhow, our bootstrap procedure can be easily adapted for CRN (Kleijnen et al, 2010).

We summarize our procedure in the following pseudocode, assuming that $\left(\mathbf{X}, \overline{\mathbf{w}}_{i}\right)$ (the original simulation $\mathrm{I} / \mathrm{O}$ data) satisfy the requirement $\min w_{i}<\max w_{i+1}$ and that classic Kriging violates the monotonicity requirement (5); moreover, for simplicity we increase the bootstrap sample size in steps of $B$ if the initial bootstrap sample of size $B$ does not give 'enough' (namely, $B^{\prime}$ ) bootstrapped monotonic Kriging metamodels:

(1) Read the original simulation $\mathrm{I} / \mathrm{O}$ data $\left(\mathbf{x}_{i}^{\prime}, \mathbf{w}_{i}^{\prime}\right)$ with $\mathbf{x}_{i}^{\prime}=\left(x_{i, 1}, \ldots, x_{i ; k}\right), \mathbf{w}_{i}^{\prime}=\left(w_{i ; 1}, \ldots, w_{i ; m_{i}}\right)$, where $i=1, \ldots, n$ and $k$ denotes the number of inputs, $n$ the number of combinations of these $k$ inputs, and $m_{i}$ the number of replicates in combination $i$.

(2) Read the bootstrap sample size $B$ and the sample size required for computing confidence intervals $B^{\prime}(\leqslant B)$; initialize the counter $b^{\prime}=0\left(b^{\prime}\right.$ denotes the actual number of monotonic bootstrapped Kriging models).

(3) Bootstrap $\left(\mathbf{x}_{i}^{\prime}, \mathbf{w}_{i}^{\prime}\right)$ (obtained in Step 1) to get the bootstrapped I/O data $\left(\mathbf{X}, \overline{\mathbf{w}}^{*}\right)$ (see the pseudo-code above (3)).

(4) Fit the Kriging model to $\left(\mathbf{X}, \overline{\mathbf{w}}^{*}\right)$ (obtained in Step 3).

(5) If the Kriging model from Step 4 is monotonic, then $b^{\prime}=b^{\prime}+1$; else proceed to the next step.

(6) Repeat Steps 3 through 5, $B$ times (where $B$ denotes the bootstrap sample size).

(7) If $b^{\prime}<B^{\prime}$, then go to Step 3 (where $B^{\prime}$ denotes the required number of monotonic bootstrapped Kriging models); else proceed to the next step.

(8) Compute confidence intervals and point estimates for the $v \geqslant 1$ 'new' input combinations, from the $B^{\prime}$ bootstrapped monotonic Kriging metamodels.

\section{$\mathrm{M} / \mathbf{M} / \mathbf{1}$ example}

In the next subsection we present some preliminary considerations for our $\mathrm{M} / \mathrm{M} / 1$ simulation; in the next subsection we present results for our monotonicitypreserving Kriging in this example.

\section{Preliminaries}

The $\mathrm{M} / \mathrm{M} / 1$ queueing model is a popular example in random simulation; for example, Ankenman et al (2010) uses this example to illustrate Kriging, and Law (2007, pp 12-47, 79-83) details the single-server queueing system, including the $\mathrm{M} / \mathrm{M} / 1$ model. Although the $\mathrm{M} / \mathrm{M} / 1$ simulation requires only very simple computer programming, its $\mathrm{I} / \mathrm{O}$ behaviour is not so simple at all-as we shall demonstrate. So this example is challenging from an analytical point of view. It is computationally inexpensive so we can easily perform extensive simulation experiments to verify our procedure. In practice, of course, simulation models often require special software for their programming, and may require much computer time; that is, the $\mathrm{M} / \mathrm{M} / 1$ model is only an illustration.

In an $\mathrm{M} / \mathrm{M} / 1$ simulation, the waiting time of customer $t$ (say) $w_{t}$ may be computed through

$$
w_{t+1}=\max \left(0, w_{t}+s_{t}-a_{t+1}\right) t=1,2, \ldots,
$$

where $w_{1}$ is determined by the initialization of the simulation run (eg, if the simulation starts in the 'empty' state, then $w_{1}=0$ ); the exponentially distributed inputs $s$ and $a$ have service rate $\mu$ and arrival rate $\lambda$. To obtain 
sampled values for these $s$ and $a$, the simulation may use a single PRN stream $p_{1}, p_{2}, \ldots$ as follows (but alternative sampling routines do exist): $s_{t}=-\ln \quad p_{2 t-1} / \mu$ and $a_{t+1}=-\ln p_{2 t} / \lambda$. The $\mathrm{M} / \mathrm{M} / 1$ model is of practical interest because it is a building block for more complicated queueing networks that are used in telecommunications, supply chains, etc. This model is also of academic interest because it generates a time series of length (say) $T$ so we have the vector output $\mathbf{w}=\left(w_{1}, \ldots, w_{T}\right)^{\prime}$ with positively correlated scalar outputs $w_{t}$; this vector may be used in steady-state analysis, and has components with nonconstant variances $\left(\operatorname{var}\left(w_{t}\right)\right.$ increases as $t$ increases, until the steady state is reached). It is well-known that various performance measures (see below) are nonlinear functions of the traffic rate (or traffic load) $x=\lambda / \mu$.

In our example we simulate this model starting not with $w_{1}=0$ (the usual initialization of Equation (7)) but with $w_{1}$ equal to its expected steady-state value. The reason is that we are not interested in the problem of determining whether the simulation has reached steady state or is still in transient state; that is, we 'cheat' and use the analytical solution for the steady-state waiting time distribution for the $\mathrm{M} / \mathrm{M} / 1$. In this way we can verify some of our simulation results; Ankenman et al (2010) use a similar trick.

We study two performance measures: (i) the steady-state mean waiting time $E\left(w_{t} \mid t \rightarrow \infty\right)=\mu_{w}$; (ii) the steady-state $90 \%$ quantile $w_{0.9}$ defined by $P\left(w_{t} \leqslant w_{0.9} \mid t \rightarrow \infty\right)=0.9$. The first measure is popular in academic research. The classic estimator of this mean is the time-series average

$$
\bar{w}=\frac{\sum_{t=1}^{T} w_{t}}{T} .
$$

The second measure (the 0.9 quantile) is more popular in practice; see Batur and Choobineh (2010), Hong (2009) and Jin et al (2003). To estimate a quantile, we sort the (autocorrelated) time series $\mathbf{w}$ from low to high - which gives the (autocorrelated) so-called 'order statistics' $w_{(1)}, \ldots, w_{(T)}$. This gives the classic point estimator of $w_{0.9}$ :

$$
\widehat{w_{0.9}}=w_{([0.9 T])} \text {. }
$$

To observe the sampling variability of the estimates of the mean and $90 \%$ quantile defined in (8) and (9), we use $m \geqslant 2$ replicates (alternative approaches are discussed in the literature; see Law, 2007, p 506). Replicate $r(r=1, \ldots, m)$ gives the average waiting time $\overline{w_{r}}$ and the estimated quantile $\widehat{w_{0.9 ;}}$. We expect these averages $\overline{w_{r}}$ to be normally distributed because of the Functional Central Limit Theorem (Lehmann, 1999). The quantile estimators $\widehat{w_{0.9} ; r}$ are only asymptotically normally distributed (Chen, 2008; Hong, 2009). We (rather arbitrarily) select a 'short' runlength $T=1000$ and a 'long' length $T=100000$, after selecting the time units for the arrival and service times such that the service rate $\mu$ equals one. Furthermore, in the preliminary investigation reported in this subsection, we
Table $1 p$ values for Kolmogorov-Smirnov test of normality

\begin{tabular}{lcccc}
\hline Runlength & \multicolumn{2}{c}{$T=1000$} & \multicolumn{2}{c}{$T=100000$} \\
\hline Traffic rate & $\lambda / \mu=0.5$ & $\lambda / \mu=0.9$ & $\lambda / \mu=0.5$ & $\lambda / \mu=0.9$ \\
Average & $<0.01$ & $<0.01$ & $>0.15$ & 0.11 \\
0.9 quantile & $<0.01$ & $<0.01$ & $>0.15$ & 0.116 \\
\hline
\end{tabular}

select only two traffic rates, $\lambda / \mu=0.5$ and $\lambda / \mu=0.9$ (in the next subsection we use more traffic rates); a high traffic rate gives stronger autocorrelation so we expect nonnormality. In this subsection, we wish to obtain accurate estimates of the true behaviour of the simulated outputs, so we select $m=1000$ replicates (our Kriging results in the next subsection will use smaller, realistic $m$ values). We test the goodness of it through the chi-square and the Kolmogorov-Smirnov tests. We present the resulting $p$-values for the latter test only, because the former test gives similar results. Table 1 shows that the estimated average and quantile are not normally distributed if the simulation run is short $(T=1000)$ - even for a relatively low traffic rate $(\lambda / \mu=0.5)$.

To evaluate the performance of our procedure, we use the following analytical results (Gross and Harris, 1998, $\mathrm{p}$ 67). The steady-state waiting time $W$ in an $\mathrm{M} / \mathrm{M} / 1$ model with traffic rate $x=\lambda / \mu$ has the distribution $P(W \leqslant w)=1-x \exp (-\mu)(1-x) w)$. This implies for the 0.9 quantile $w_{0.9}: \quad 1-x \quad \exp \left(-\mu(1-x) w_{0.9}\right)=0.9$, so $w_{0.9}=-\ln (0.1 / x) / \mu(1-x)$. The mean $\mu_{w}$ is $x /[\mu(1-x)]$.

\section{Results for monotonicity-preserving Kriging}

In practice, a small number of replicates $m$ is used if a single simulation run takes much computer time; nevertheless, $m$ should be large enough to obtain adequate signal/noise. This signal/noise criterion does not give a magic number of observations, but should guide preliminary experimentation (also see the call-centre example of simulation optimization through Kriging, detailed in Kleijnen et al, 2010). In our $\mathrm{M} / \mathrm{M} / 1$ example the signal/ noise depends on the runlength $T$. After some experimentation, we select $T=1000$ and $m=5$ for our $\mathrm{M} / \mathrm{M} / 1$ example. Furthermore, we select $n=5$ values for the traffic rate $\lambda / \mu$ (for higher $n$ values, we do not expect wiggly behaviour so there is no need to bootstrap for monotonicity preservation). We select these $n$ points such that the traffic rates $x_{i}=\lambda_{i} / \mu_{i}$ are inside the experimental area $0.1 \leqslant x \leqslant 0.9$. We select a bootstrap sample size $B=100$; sometimes (in 5 of the 100 'macroreplicates', defined above Equation (14)), this does not give enough monotonicitypreserving Kriging models (so $B^{\prime}<100$ ), so we bootstrap another $B=100$ times. We assume that the users are interested in either the mean or the $90 \%$ quantile, so we 
bootstrap the estimated mean and quantile independently (ie, we do not resample the $m$ correlated pairs $\left(\overline{w_{r}}, \widehat{w_{0.9} r}\right)$ ).

Besides the $n=5$ old points, we (rather arbitrarily) select $v=25$ new points that are to be predicted. We select these new points such that no extrapolation is needed, because Kriging metamodels are known to be poor extrapolators; see Antognini and Zagoraiou (2010). To select specific new points, we use LHS.

To estimate whether our point predictor (the bootstrap median) for the true output (say) $\zeta$ is better than the classic Kriging predictor $y$, we estimate the Integrated MSE (the average over all test points) through

$$
\begin{aligned}
\overparen{M S} E^{*} & =\frac{\sum_{u=1}^{v}\left(y_{u ;\left(\left[0.50 B^{\prime}\right\rceil\right)}^{*}-\zeta_{u}\right)^{2}}{v} ; \\
\widehat{I M S E} E & =\frac{\sum_{u=1}^{v}\left(y_{u}-\zeta_{u}\right)^{2}}{v},
\end{aligned}
$$

assuming that we know the true output $\zeta_{u}-$ as is the case for the $\mathrm{M} / \mathrm{M} / 1$ example. Notice that we use the same bootstrapped Kriging metamodel to obtain point predictors for the $v$ different test points.

To estimate whether our confidence interval gives better coverage than the classic Kriging confidence interval does, we compute the indicator function

$$
\begin{gathered}
I_{u}^{*}=1 \text { if } y_{u ;\left(\left[0.50 B^{\prime}\right\rceil\right)}^{*}<\zeta_{u}<y_{u ;\left(\left[0.95 B^{\prime}\right\rceil\right)}^{*} ; \\
\text { else } I^{*}=0
\end{gathered}
$$

for our bootstrap procedure (using the well-known percentile method; see Efron and Tibshirani, 1993, p 170). For classic Kriging we compute

$$
\begin{gathered}
I_{u}=1 \text { if } y_{u}-1.64 \widehat{\sigma}_{y_{u}}<\zeta_{u}<y_{u}+1.64 \widehat{\sigma}_{y_{u}} ; \\
\text { else } I=0
\end{gathered}
$$

where $\widehat{\sigma_{y_{u}}^{2}}$ is the estimated predictor variance provided by the classic Kriging literature and software, including DACE. Unlike our bootstrap interval (11), the classic interval (12) is symmetric around its point estimate $y_{t}$ and may include negative values - even if negative values are impossible, as is the case for waiting times. Analogously to (10), we estimate the coverage averaged over all $v$ test points:

$$
\overline{I^{*}}=\frac{\sum_{u=1}^{v} I_{u}^{*}}{v} ; \bar{I}=\frac{\sum_{u=1}^{v} I_{u}}{v} .
$$

In our $\mathrm{M} / \mathrm{M} / 1$ example, we obtain (say) $L$ so-called 'macro-replicates', which differ only in their PRN seeds. Because of (13), we let $\overline{I_{l}^{*}}$ denote the average indicator for our bootstrap predictor in macro-replicate $l$, and $\overline{I_{l}}$ the analogue for the classic predictor. Our predictor then has the better coverage if the macro-replicates give an average $\overline{\overline{I^{*}}}=\sum_{l} \overline{I_{l}^{*}} / L$ that is closer to the nominal value 0.90 than the classic predictor's $\overline{\bar{I}}=\sum_{l} \bar{I}_{l} / L$.
We decide to obtain $L=100$ macro-replicates. From these macro-replicates we estimate a $90 \%$ confidence interval for the IMSE in classic Kriging, in the standard way:

$$
\overline{I \widehat{M S} E} \pm 1.64 \frac{S(I \widehat{M S} E)}{\sqrt{L}}
$$

where

$$
\begin{aligned}
& \widehat{I \widehat{M S E}}=\frac{\sum_{l=1}^{L} I \widehat{M S} E_{l}}{L} ; \\
& S(\widehat{I M S} E)=\sqrt{\frac{\sum_{l=1}^{L}\left(I \widehat{M S} E_{l}-\widehat{I \widehat{M S} E}\right)^{2}}{L-1} .} .
\end{aligned}
$$

For our monotonicity-preserving bootstrap Kriging, we use analogous formulas. For the coverage and the length of the confidence interval we also use analogous formulas.

In some macro-replicates the classic Kriging metamodels are monotonic, so there is no need to bootstrap. To check whether a classic Kriging metamodel gives a monotonic $\mathrm{I} / \mathrm{O}$ function, we check whether all the gradients estimated at the $n$ old points are positive: $\forall i: \nabla y_{i} \geqslant \mathbf{0}(i=1, \ldots, n)$; also see (6). If a macro-replicate satisfies this condition, then we sample a new macro-replicate; we stop after we have $L=100$ macro-replicates with nonmonotonic classic Kriging metamodels.

Figure 1 shows a macro-replicate in which the classic Kriging meta-model shows wiggling, whereas a bootstrapped model is monotonic. This figure also showsfor each of the $n=5$ input values - the $m=5$ replicated simulation outputs (see dots) and their averages (see stars). Furthermore, the figure shows the analytical (dotted) I/O curve. Notice that for low traffic rates the variability of the individual simulation outputs is so small that this variability is hardly visible; nevertheless, the bootstrap finds a monotonic Kriging model. (The data of all figures in this article, and the corresponding Matlab code, are available from the authors.)

Wiggling means that the derivative $d \widehat{y} / d x$ is negative for at least one $x$ value in the area of interest. Wiggling may also occur at new points (besides the old points; see Figure 1). In the $\mathrm{M} / \mathrm{M} / 1$ example (which has a single input), we check whether Kriging gives wiggling at 100 new points, spread uniformly across the experimental range. (In applications with multiple inputs, however, such a grid search is rather expensive, so we may find the $\mathbf{x}$ point that minimizes the components of the estimated gradient $\nabla \widehat{y}$; if at least one component is negative, then the Kriging predictor $\widehat{y}$ shows wiggling so we apply bootstrapping.)

Note: We also apply Universal Kriging, which replaces the constant term in Ordinary Kriging by a first-order and a second-order polynomial respectively (see the Online Appendix). This Universal Kriging, however, turns out not to remove the wiggling, so we focus on Ordinary Kriging. 


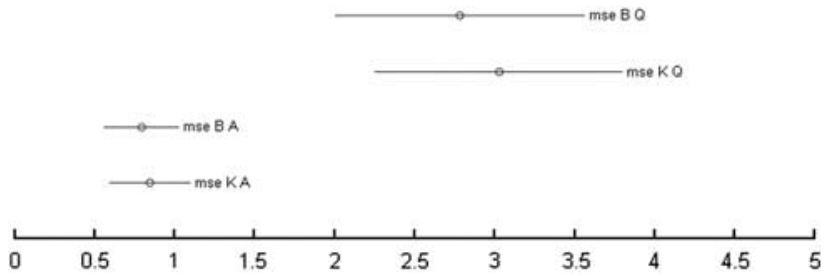

Figure 2 Estimated IMSE of classic and monotonic bootstrapped Kriging for the mean and $90 \%$ quantile, with $n=5$, $m=5, T=1000$.

Figure 2 gives the estimated IMSE for the average and the $90 \%$ quantile; the symbol 'KA' stands for 'Kriging the Average', 'BQ' for 'Bootstrapping the Quantile', etc. Classic Kriging uses the MSE as the criterion when optimizing the Kriging weights; so when we impose the monotonicity constraint, we do not expect significantly lower MSE. This figure shows that our bootstrap gives smaller estimated IMSEs, albeit not significantly smaller; of course, the $90 \%$ quantile has larger IMSEs than the mean has.

Figure 3 gives the estimated coverage for the average and the $90 \%$ quantile. This figure shows that our bootstrap gives significantly higher estimated coverages for the mean and the quantile. Unfortunately, all estimated coverages are significantly lower than the nominal value of $90 \%$; we shall return to this problem.

Figure 4 gives the corresponding estimated widths. This figure shows that our bootstrap gives widths that are not significantly shorter; see the point estimate. The variability of the width is smaller for our bootstrapped Kriging; see the length of the confidence interval for the width. Together the latter two figures show that our bootstrap gives better coverage without lengthening the confidence interval.

Because the coverage is significantly lower than the prespecified nominal value of $90 \%$, we repeat our experiment with second-order polynomial (linear regression) metamodels, estimated through Ordinary Least Squares (OLS). This gives coverages for the mean and the quantile that are lower than Kriging (classic or bootstrapped) does. The variability of the coverage (measured by the length of the confidence interval for the estimated coverage) is smaller for the second-order polynomial; we conjecture that this phenomenon arises because in this metamodel only the three regression coefficients may vary. Details are given in the Online Appendix.

To further examine this low coverage (of the Kriging metamodels for $\mathrm{M} / \mathrm{M} / 1$ simulation models), we increase $n$ coverage

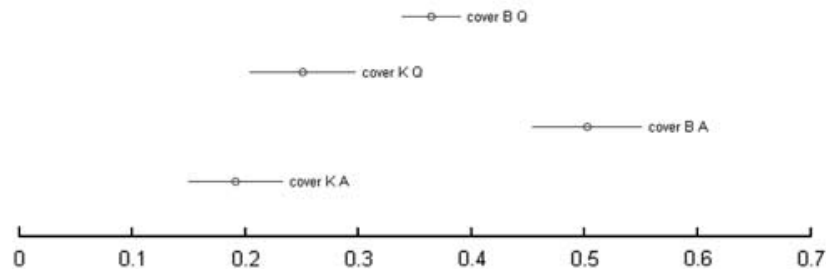

Figure 3 Coverage of classic and monotonicity-preserving bootstrapped Kriging for the mean and $90 \%$ quantile, with $n=5, m=5, T=1000$.

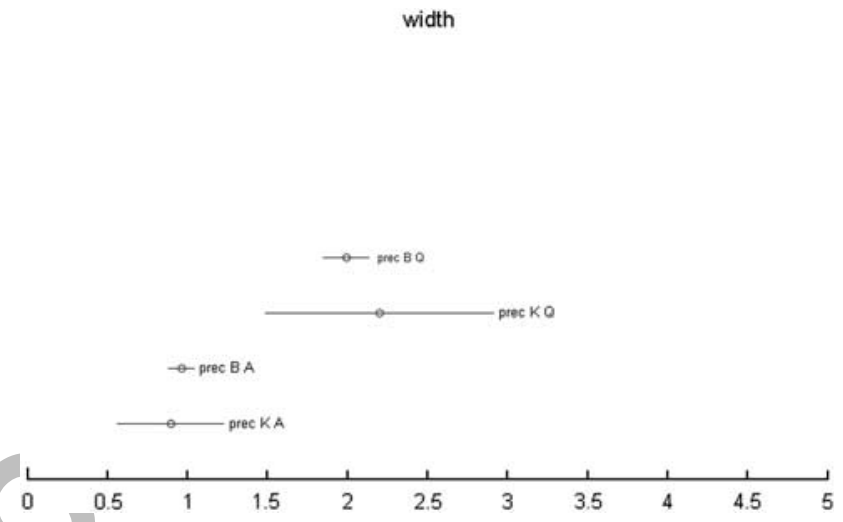

Figure 4 Width of confidence interval for classic and monotonicity preserving bootstrapped Kriging for the mean and $90 \%$ quantile, for $n=5, m=5, T=1000$.

(number of old points) from 5 to 10 (we still limit our analysis to the $L=100$ macro-replicates that show nonmonotonic classic Kriging metamodels). Table 2 shows that such a larger number of input combinations increases the estimated coverages for both classic Kriging and monotonicity-preserving bootstrapped Kriging; this improved coverage is explained by the better fit of the metamodel resulting from an 'adequate' sample size; also see Loeppky et al (2009), who suggest that a valid Kriging metamodel requires $n=10 k$ (which in our example implies $n=10$ ). These coverages are close to the nominal $90 \%$ for our monotonicity-preserving bootstrapped Kriging, whereas classic Kriging still gives coverages far below the desired nominal value. Our improved coverage does not require significantly longer confidence intervals.

\section{Conclusions and future research}

In practice, simulation may be computationally expensive, so only a few input combinations are simulated and these 
Table 2 Coverage in classic Kriging $(K)$ and monotonicitypreserving bootstrapped Kriging $(B)$, for the Average $(A)$ and the $90 \%$ Quantile $(Q)$, with $n=10, m=5, T=1000$

\begin{tabular}{lccc}
\hline & Lower bound & Median & Upper bound \\
\hline Coverage KA & 0.226 & 0.353 & 0.449 \\
Coverage BA & 0.831 & 0.845 & 0.858 \\
Coverage KQ & 0.331 & 0.442 & 0.552 \\
Coverage BQ & 0.830 & 0.844 & 0.859 \\
Width KA & 0.233 & 0.306 & 0.379 \\
Width BA & 0.371 & 0.382 & 0.392 \\
Width KQ & 1.023 & 1.047 & 1.070 \\
Width BQ & 0.948 & 1.208 & 1.477 \\
\hline
\end{tabular}

few combinations are replicated only a few times (small $n$ and $m$ ). In such situations, classic Kriging may give nonmonotonic metamodels, which contradict the prior qualitative knowledge of the simulation analysts and their clients.

The clients may then reject the metamodel, and the analysts may find the metamodel to give too low coverage. Our simple monotonicity-preserving bootstrapped Kriging may improve the results; that is, we may obtain better coverage without longer confidence interval. Unfortunately, these coverages may still be lower than desired, because the small number of simulation observations may give too little information to estimate an adequate metamodel - be that metamodel a classic Kriging model, a monotonicity-preserving bootstrapped Kriging model, a polynomial regression model, etc. In such situations we would advise the analysts to spend more computer time in order to obtain reliable results. But while awaiting these results, the analysts can bootstrap the too small sample to obtain a monotonicity-preserving bootstrapped Kriging metamodel that is better than the classic Kriging or the regression metamodel.

An additional advantage of our bootstrap Kriging is that our confidence interval (11) does not include negative values if negative values are impossible. Moreover, our Kriging metamodel is not an exact interpolator, which is attractive because the average simulation outputs still show sampling variation.

We point out that it does not take much computer time to apply bootstrap Kriging to the small $\mathrm{I} / \mathrm{O}$ data set that consists of the 'original' simulation $\mathrm{I} / \mathrm{O}$ data. To obtain those simulation outputs may take weeks on a supercomputer. Resampling those data to obtain $\bar{w}_{i}^{*}$ takes only microseconds. To fit a Kriging metamodel to the $(n \times k) \mathrm{I} / \mathrm{O}$ bootstrapped data set $\left(\mathbf{X}, \overline{\mathbf{w}}^{*}\right)$ takes no more than a few minutes on a simple PC, because only small matrices need to be inverted. So if the simulation's $\mathrm{I} / \mathrm{O}$ function is known to be monotonic, then our search for $B^{\prime}$ with $1 \ll B^{\prime} \ll B$ monotonicity-preserving bootstrapped Kriging metamodels will be successful.
If the analysts are not certain that the simulation model implies a monotonic I/O function, then they may apply both classic and bootstrapped Kriging and compare the MSEs for a few test points to decide which Kriging variant to use.

In future research we may investigate the following topics. Our monotonicity-preserving bootstrap procedure may be applied to more complicated simulation models; for example, supply chains. Our procedure may also be applied to 'stochastic Kriging' metamodels (Yin et al, 2009; Ankenman et al, 2010). We may also use bootstrapped Kriging to preserve other known characteristics of the $\mathrm{I} / \mathrm{O}$ function besides monotonicity; namely, convexity, nonnegativeness, etc. Finally, we may apply our bootstrap to other metamodelling methods besides Kriging; for example, isotonic regression (Dette et al, 2006; Lim and Glynn, 2006).

In general, the bootstrap is a very versatile statistical technique that may be used to analyse nonlinear and non-Gaussian statistics; Efron and Tibshirani (1993) give advanced theory and many applications. We ourselves have applied bootstrapping to a large variety of problems; for example, optimization of simulated systems, validation of metamodels and simulation models, and ranking of scientific journals.

Acknowledgements - We thank two (anonymous) referees and Dick den Hertog (Tilburg University) for their comments on earlier versions, which lead to drastic revisions.

\section{References}

Ankenman B, Nelson B and Staum J (2010). Stochastic Kriging for simulation metamodeling. Operations Research 58(2): 371-382.

Antognini B and Zagoraiou M (2010). Exact optimal designs for computer experiments via Kriging metamodelling. Journal of Statistical Planning and Inference 140(9): 2607-2617.

Batur D and Choobineh F (2010). A quantile-based approach to system selection. European Journal of Operational Research 202(3): 764-772.

Chen EJ (2008). Some procedures of selecting the best designs with respect to quantile. Simulation 84: 275-284.

Chen X, Ankenman B and Nelson BL (2010). The effects of common random numbers on stochastic Kriging metamodels. Working Paper, Department of Industrial Engineering and Management Sciences, Northwestern University, Evanston, IL USA

Den Hertog D, Kleijnen JPC and Siem AYD (2006). The correct Kriging variance estimated by bootstrapping. Journal of the Operational Research Society 57(4): 400-409.

Dette H, Neumeyer N and Pilz KF (2006). A simple nonparametric estimator of a strictly monotone regression function. Bernoulli 12(3): 469-490.

Efron B and Tibshirani RJ (1993). An Introduction to the Bootstrap. Chapman \& Hall: New York.

Feelders AJ (2000). Prior knowledge in economic applications of data mining. Proceedings of the Fourth European Conference on 
Principles and Practice of Knowledge Discovery in Databases, Springer: New York, pp 395-400.

Forrester A, Sobester A and Keane A (2008). Engineering Design via Surrogate Modelling: A Practical Guide. Wiley: Chichester, UK.

Frazier P, Powell W and Dayanik S (2009). The knowledgegradient policy for correlated normal beliefs. INFORMS Journal on Computing 21: 599-613.

Gross D and Harris CM (1998). Fundamentals of Queueing Theory. Wiley: New York.

Hong LJ (2009). Estimating quantile sensitivities. Operations Research 57(1): 118-130.

Jin X, Fu MC and Xiong X (2003). Probabilistic error bounds for simulation quantile estimators. Management Science 14(2): 230-246.

Kleijnen JPC (2008). Design and Analysis of Simulation Experiments. Springer Science + Business Media, New York.

Kleijnen JPC (2009). Factor screening in simulation experiments: Review of sequential bifurcation. In: Alexopoulos C, Goldsman $\mathrm{D}$ and Wilson JR (eds). Advancing the Frontiers of Simulation: A Festschrift in Honor of George S. Fishman. Springer: New York, pp 169-173.

Kleijnen JPC, van Beers W and van Nieuwenhuyse I (2010). Constrained optimization in expensive simulation: Novel approach. European Journal of Operational Research 202: 164-174.

Law AM (2007). Simulation Modeling and Analysis. Fourth edn, McGraw-Hill: Boston.

Lehmann EL (1999). Elements of Large-Sample Theory. Springer: New York.

Lim E and Glynn PW (2006). Simulation-based response surface computation in the presence of monotonicity. In: Perrone LF, Wieland FP, Liu J, Lawson BG, Nicol DM and Fujimoto RM (eds). Proceedings of the 2006 Winter Simulation Conference, The Society for Computer Simulation International (SCS): San Diego, CA, pp 264-271.
Loeppky JL, Sacks J and Welch W (2009). Choosing the sample size of a computer experiment: A practical guide. Technometrics 51: 366-376.

Lophaven SN, Nielsen HB and Sondergaard J (2002). DACE: $a$ Matlab Kriging Toolbox, version 2.0. IMM Technical University of Denmark: Lyngby.

Sacks J, Welch WJ, Mitchell TJ and Wynn HP (1989). Design and analysis of computer experiments. Statistical Science 4(4): $409-435$.

Santner TJ, Williams BJ and Notz WI (2003). The Design and Analysis of Computer Experiments. Springer-Verlag: New York.

Siem AYD (2007). Property preservation and quality measures in meta-models. PhD dissertation, Tilburg University, Tilburg, the Netherlands.

Simpson TW, Booker AJ, Ghosh D, Giunta AA, Koch PN and Yang R-J (2004). Approximation methods in multidisciplinary analysis and optimization: A panel discussion. Structural and Multidisciplinary Optimization 27(5): 302-313.

Van Beers W and Kleijnen JPC (2003). Kriging for interpolation in random simulation. Journal of the Operational Research Society 54(3): 255-262.

Velikova M (2006). Monotone models for prediction in data mining. $\mathrm{PhD}$ dissertation, Tilburg University, Tilburg, the Netherlands.

Yin $\mathrm{J}, \mathrm{Ng} \mathrm{SH}$ and $\mathrm{Ng} \mathrm{KM} \mathrm{(2009).} \mathrm{A} \mathrm{study} \mathrm{on} \mathrm{the} \mathrm{effects} \mathrm{of}$ parameter estimation on Kriging model's prediction error in stochastic simulations. In: Rossini MD, Hill RR, Johansson B, Dunkin A and Ingalls RG (eds). Proceedings of the 2009 Winter Simulation Conference, The Society for Computer Simulation International (SCS): San Diego, CA, pp 674-685.

Received June 2010; accepted November 2011 after two revisions

Supplementary Information accompanies the paper on Journal of the Operational Research Society website (http:// www.palgrave-journals.com/jors/) 